\title{
New records of Dolichopodidae (Diptera) from Russian Primorye and notes on some Chinese species
}

\author{
Новые находки Dolichopodidae (Diptera) в Приморском крае \\ России и заметки о некоторых видах из Китая
}

\author{
I.Ya. Grichanov \\ И.Я. Гричанов
}

\begin{abstract}
All-Russian Institute of Plant Protection, Podbelskiy roadway 3, St. Petersburg-Pushkin 196608, Russia. E-mail: grichanov@mail.ru Всероссийский институт защиты растений, шоссе Подбельского 3, Санкт-Петербург-Пушкин 196608, Россия.
\end{abstract}

KEY WORDS: Dolichopodidae, Russia, Primorsky Territory, new records.

КЛЮЧЕВЫЕ СЛОВА: Dolichopodidae, Россия, Приморский край, новые указания.

ABSTRACT. A new material of Dolichopodidae has been recently found and identified, and includes 53 species; five species are found for the first time in Russia (Amblypsilopus janatus, Argyra arrogans, Dolichopus aubertini, Medetera flavigena, Micromorphus jinshanensis), and three species in the Primorsky Territory ( $D i-$ aphorus nigricans, Dolichopus punctum, Neurigona micropyga). In total, nearly 170 species are recorded from Russian Primorye that apparently makes up $80-90 \%$ of actual Dolichopodidae fauna of this Territory. All Russian and Chinese species of the Hercostomus cyaneculus group are transferred to the genus Poecilobothrus Mik, 1878 (comb. n.): P. arcticus (Yang, 1996), P. brevipilosus (Yang et Saigusa, 2002), P. brunus (Wei, 1997), $P$. cucullus (Wei, 1997), P. cyaneculus (Wei, 1997), P. flaveolus (Negrobov et Chalaya, 1987), P. lii (Yang, 1996), P. longipilosus (Yang et Saigusa, 2001), P. luchunensis (Yang et Saigusa, 2001), P. mentougouensis (Zhang, Yang et Grootaert, 2003), P. palustrus (Wei, 2006), P. potanini (Stackelberg, 1934), P. pterostichoides (Stackelberg, 1934), P. saetosus (Yang et Saigusa, 2002), P. singularis (Yang et Saigusa, 2001), P. zhejiangensis (Yang, 1996). Poecilobothrus brevipilosus is synonymized with $P$. pterostichoides (syn.n.). Poecilobothrus flavifemoratus Grichanov et Tonguc, 2010, subspecies of $P$. varicoloris (Becker, 1917) is raised to a species level (stat.n.). This paper provides also distribution pattern for each collected species.

РЕЗЮМЕ. Новый материал по семейству Dolichopodidae Приморского края обнаружен и определён. Новые указания включают 53 вида, в том числе пять видов, новых для фауны России (Amblypsilopus janatus, Argyra arrogans, Dolichopus aubertini, Medetera flavigena, Micromorphus jinshanensis), и три вида, новых для Приморского края (Diaphorus nigricans,
Dolichopus punctum, Neurigona micropyga). Всего в крае отмечено почти 170 видов, что, по-видимому, составляет 80-90\% приморской фауны Dolichopodidae. Bce виды группы Hercostomus cyaneculus перенесены в род Poecilobothrus Mik, 1878 (comb. n.): $P$. arcticus (Yang, 1996), P. brevipilosus (Yang et Saigusa, 2002), P. brunus (Wei, 1997), P. cucullus (Wei, 1997), P. cyaneculus (Wei, 1997), P.flaveolus (Negrobov et Chalaya, 1987), P. lii (Yang, 1996), P. longipilosus (Yang et Saigusa, 2001), P. luchunensis (Yang et Saigusa, 2001), P. mentougouensis (Zhang, Yang et Grootaert, 2003), P. palustrus (Wei, 2006), P. potanini (Stackelberg, 1934), P. pterostichoides (Stackelberg, 1934), P. saetosus (Yang et Saigusa, 2002), P. singularis (Yang et Saigusa, 2001), P. zhejiangensis (Yang, 1996). Poecilobothrus brevipilosus помещён в синонимы к $P$. pterostichoides (syn.n.). Poecilobothrus flavifemoratus Grichanov et Tonguc, 2010, подвид P. varicoloris (Becker, 1917) признан видом (stat.n.). В статье приведено также общее распространение для каждого вида.

\section{Introduction}

Primorsky Territory, or Primorye, is located in the southeasternmost region of the Russian Far East, bordering by China (Jilin and Heilongjiang), North Korea, Khabarovsk Territory and waters of the Sea of Japan. Most of the territory is mountainous with almost $80 \%$ of it forested. There are mountainous tundra areas, conifers and coniferous-deciduous forests, and forest-steppe [Kiselev, Kudryavtseva, 1998].

The first information about Primorian long-legged flies was published since 1928 in a series of works of the famous Soviet dipterologist A.A. Stackelberg (Leningrad, the Zoological Institute - ZIN) who treated Diptera material collected during the 1927 Far-Eastern Hydro-

How to cite this article: Grichanov I.Ya. 2020. New records of Dolichopodidae (Diptera) from Russian Primorye and notes on some Chinese species // Russian Entomol. J. Vol.29. No.4. P.432-438. doi: 10.15298/rusentj.29.4.12 
graphic expedition of the Academy of Sciences of the USSR. The results of treatment of rich Dolichopus materials collected by the 1937, 1940 and 1946 Russian expeditions were published by the well known entomologist E.S. Smirnov (the Moscow State University). Since 1950s, the dolichopodid collections of ZIN, Zoological Museums of Moscow State University (ZMUM) and Voronezh State University (VSU) replenished by various expeditions to Primorye. These materials were used in a number of taxonomical revisions and faunistic reviews of dolichopodid fauna of the USSR and Russia published by O.P. Negrobov (VSU) and his disciples since 1960s.

Recently, new dolichopodid material was collected by ZIN and ZMUM expeditions to Primorye, and several older specimens were identified. The very latest identification keys published for different genera of the Palaearctic, Russian, Chinese or Japanese faunas were used. The results of the species treatment are presented below. A new material of Dolichopodidae includes 53 species; five species are found for the first time in Russia, and three species in the Primorsky Territory. In total, nearly 170 species are recorded now from Russian Primorye that apparently makes up $80-90 \%$ of actual Dolichopodidae fauna of this Territory. The information on the number of species within genera and on the global distribution for each species collected follows Negrobov et al. [2013], Grichanov [2017] and Yang et al. [2018]. The type localities are provided and the country lists are arranged alphabetically. The words "Region" (oblast) and "Territory" (kray) are omitted from the list of Russian regions. Remarks are provided where deemed necessary. The collected material of the newly-recorded species is mounted on pins except the flies collected by O. Kosheleva and kept in ethanol.

\section{New Records}

\section{Genus Amblypsilopus Bigot, 1888}

NOTES. Two species of this mainly tropical genus are known from Russian Primorye. One species was originally described from this territory. The Chinese fauna numbers 45 species with possible inclusion of the Russian Amblypsilopus species.

\section{Amblypsilopus janatus (Negrobov, 1984)}

MATERIAL EXAMINED. $20^{7}, 2 \circ$, Khasan distr., Andreevka, MES [=Marine Experimental Station], 7.08.1978, Kasparyan [ZIN]; $10^{7}$, Posiet, seashore, $42.67^{\circ} \mathrm{N}, 130.82^{\circ} \mathrm{E}, 31.07 .2018$, N. Vikhrev [ZMUM]; $20^{\top}$, Vladivostok env., $43.229^{\circ} \mathrm{N}, 131.975^{\circ} \mathrm{E}, 25.07 .2019$, E. Erofeeva [ZMUM].

DISTRIBUTION. Type locality: Japan: Ofune. Palaearctic: Japan. New for Russia and Primorye.

Genus Argyra Macquart, 1834

NOTES. Six species of Argyra are known from Primorye. Four species were originally described from this territory. The Chinese fauna numbers 9 species.

Argyra arrogans Takagi, 1960

MATERIAL EXAMINED. $10^{7}$, Khanka Lake, $45.06^{\circ} \mathrm{N}$, 131.99 ${ }^{\circ} \mathrm{E}, 15-19.06 .2014$, N. Vikhrev [ZMUM].

DISTRIBUTION. Type locality: Japan: Hokkaido, Aizan-
Kei. Palaearctic: Japan; Oriental: China (Zhejiang, Guizhou). New for Russia and Primorye.

Argyra flavida Negrobov, 1973

MATERIAL EXAMINED. $10^{7}, 10 \mathrm{~km}$ NE Vladivostok, $43.21^{\circ} \mathrm{N}, 132.07^{\circ} \mathrm{E}, 21-29.07 .2019$, E. Erofeeva [ZMUM].

DISTRIBUTION. Type locality: Russia: Primorye, Spasskii, Yakovlevka. Palaearctic: Russia (Primorye).

Genus Asyndetus Loew, 1869

NOTES. Two endemic species of closely related genera Asyndetus and Cryptophleps Lichtwardt, 1898 are known from Primorye. Fourteen species are known from China.

Asyndetus diaphoriformis

Negrobov et Shamshev, 1986

MATERIAL EXAMINED. $10^{7}$, Kamenushka, $43.62^{\circ} \mathrm{N}$, 132.23ํㅡ, 22-24.06.2014, N. Vikhrev [ZMUM]

DISTRIBUTION. Type locality: Russia: Primorye, Kedrovaya Pad Reserve. Palaearctic: Russia (Primorye).

Genus Campsicnemus Haliday, 1851

NOTES. Seven species of Campsicnemus are known from Primorye. Five species were originally described from this territory. The Chinese fauna numbers five species.

\section{Campsicnemus unipunctatus \\ Negrobov et Zlobin, 1978}

MATERIAL EXAMINED. $30^{7}$, Ussuri distr., Gornotaezhnoe, 15.07.1980, V. Zlobin [ZIN].

DISTRIBUTION. Type locality: Russia: Maritime Territory, 57 km W Ternei river, Sikhote-Alinsky Reserve. Palaearctic: Russia (Primorye).

\section{Genus Chrysotimus Loew, 1857}

NOTES. Two species of the genus are known from Primorye. Thirty seven species are known from China. None species was reported from the neighboring Chinese provinces.

Chrysotimus flavisetus Negrobov, 1978

MATERIAL EXAMINED. $20^{7}$, Khanka Lake, $43.13^{\circ} \mathrm{N}$, 132.80 ${ }^{\circ}, 21-24.07 .2018$, N. Vikhrev [ZMUM].

DISTRIBUTION. Type locality: Russia: Maritime Territory, Sichote-Alinsky Reserve, $37 \mathrm{~km}$ from Ternei, river Serebryanka. Palaearctic: Russia (Primorye).

Chrysotimus spinuliferus Negrobov, 1978

MATERIAL EXAMINED. 10, 19 , Ussuri distr., Gornotaezhnoe, 15.07.1980, V. Zlobin [ZIN]; 30', 19, Bikin River, $22 \mathrm{~km}$ upstream Svetlovodnaya River mouth, 15.07.1980, V. Zlobin [ZIN]; 107, Anisimovka, 18.08.2004, V.Zlobin [ZIN]; $30^{\top}$, Ryazanovka River, $46.827^{\circ} \mathrm{N}$, $131.23^{\circ} \mathrm{E}, 1.08 .2018$, N. Vikhrev [ZMUM]; $20^{\top}$, Khanka Lake, $45.825^{\circ} \mathrm{N}, 131.02^{\circ} \mathrm{E}, 15-19.07 .2018$, N. Vikhrev [ZMUM].

DISTRIBUTION. Type locality: Russia: Maritime Territory, Yakovlevka. Palaearctic: Russia (Primorye, Sakhalin, Yakutia).

Genus Chrysotus Meigen, 1824

NOTES. Several more species collected from the South of the Primorsky Territory are found in ZMUM collection. They need further study, because the Chinese fauna numbers more than 200 described species.

\section{Chrysotus degener Frey, 1917}

MATERIAL EXAMINED. $10^{7}$, Khankaisky distr., $6 \mathrm{~km} \mathrm{~N}$ Novokachalinsk, $45^{\circ} 09^{\prime} 31.8^{\prime \prime} \mathrm{N}, 131^{\circ} 59^{\prime} 58.2^{\prime \prime} \mathrm{E}, 31.08-4.09 .2019$, Lake shore, Kosheleva [ZIN]

DISTRIBUTION. Type locality: Sri Lanka: Anurad- 
hapura. Palaearctic: China (Anhui, Beijing, Heilongjiang, Jiangsu, Liaoning, Shaanxi), Russia (Amur Region, Primorye, Yakutia); Oriental: China (Chongqing, Guangxi, Henan, Taiwan, Yunnan, Zhejiang), India, Myanmar, Pakistan, Sri Lanka.

\section{Genus Diaphorus Meigen, 1824:32 \\ Diaphorus nigricans Meigen, 1824}

MATERIAL EXAMINED. $10^{\top}$, Kamenushka, $43.62^{\circ} \mathrm{N}, 132.23^{\circ} \mathrm{E}$, 22-24.06.2014, N. Vikhrev [ZMUM]. 10 , Luchegorsk, $46.47^{\circ} \mathrm{N}$ $134.32^{\circ} \mathrm{E}, 22-24.06 .2014, \mathrm{~N}$. Vikhrev [ZMUM]. $10^{\top}$, Ryazanovka River, $46.827^{\circ} \mathrm{N}, 131.23^{\circ} \mathrm{E}, 1.08 .2018$, N. Vikhrev [ZMUM].

DISTRIBUTION. Type locality: Germany. Palaearctic: Abkhazia, Austria, Belarus, Belgium, China (Xinjiang), Czech, Denmark, Estonia, Finland, France, Germany, Greece, Hungary, India (Kashmir), Ireland, Italy, Israel, Netherlands, Norway, Poland, Romania, Russia (Amur Region, Karelia, Krasnodar, Leningrad, Moscow, Murmansk, Yakutia), Spain, Sweden, Switzerland, UK; Afrotropical, Oriental, Nearctic and Neotropical Regions. New for Primorye.

\section{Diaphorus ozerovi Selivanova,}

Negrobov et Maslova, 2011

MATERIAL EXAMINED. $10^{7}$, Andreevka, $42.7^{\circ} \mathrm{N}, 131.1^{\circ} \mathrm{E}$, 26-31.07.2018, N. Vikhrev [ZMUM].

DISTRIBUTION. Type locality: Russia: Primorye, Suchan. Palaearctic: Russia (Primorye).

\section{Diaphorus parenti Stackelberg, 1928}

MATERIAL EXAMINED. $10^{7}$, Andreevka, $42.64^{\circ} \mathrm{N}, 131.13^{\circ} \mathrm{E}$, 25-30.06.2014, N. Vikhrev [ZMUM]; $10^{7}$, Anuchino env., $43.95^{\circ} \mathrm{N}$, 133.05 ${ }^{\circ} \mathrm{E}, 20-21.06 .2014, \mathrm{~N}$. Vikhrev [ZMUM].

DISTRIBUTION. Type locality: Russia: Tigrovaya, Suchansky District, Primorsky Territory (originally published as "Siberia orientalis: prov. Transbaikalica, flumen Antipicha, pro oppidum Tshita; prov. Litoralis distr. Sutshanicus; Sedanka distr. Vladivostok". Palaearctic: China (Ningxia, Hebei, Henan), Russia (Chita, Karachai-Cherkessia, Primorye).

\section{Genus Dolichopus Latreille, 1796}

\section{Dolichopus agilis Meigen, 1824}

MATERIAL EXAMINED. 207, Kamenushka, 29.08.1978, V. Zlobin [ZIN].

DISTRIBUTION. Type locality: not given. Palaearctic: Andorra, Austria, Belgium, Bosnia and Herzegovina, Czech, China (Hebei, Inner Mongolia, Ningxia, Gansu), Denmark, Estonia, France, Germany, Hungary, Italy, Kazakhstan, Poland, Netherlands, Slovakia, Sweden, Switzerland, Russia (Altai Rep., Amur Region, Buryatia, Irkutsk, Kuril Is., Novosibirsk, Sayan Mts., Primorye, Yakutia), UK, Ukraine.

\section{Dolichopus albipalpus Negrobov, 1973}

MATERIAL EXAMINED. 10", Anisimovka, 11.07.2004, V. Zlobin [ZIN].

DISTRIBUTION. Type locality: Mongolia: "Central aimak, Zaisan, sudlich von BergBogdo ul.”. Palaearctic: Russia (Primorye), Mongolia.

\section{Dolichopus aubertini Parent, 1934}

MATERIAL EXAMINED. 20, Khanka Lake, $45.06^{\circ} \mathrm{N}$, $131.99^{\circ} \mathrm{E}, 15-19.06 .2014, \mathrm{~N}$. Vikhrev [ZMUM]; $10^{\top}$, Andreevka, $42.64^{\circ} \mathrm{N}, 131.13^{\circ} \mathrm{E}, 25-30.06 .2014$, N. Vikhrev [ZMUM].

DISTRIBUTION. Type locality: China: "Tien-tsin" [=Tianjin]. Palaearctic: China (Hebei, Beijing, Tianjin). New for Russia and Primorye.
Dolichopus bianchii Stackelberg, 1929

MATERIAL EXAMINED. 10, Udege Legend National park, $45^{\circ} 46^{\prime} 05.2^{\prime \prime} \mathrm{N}, 135^{\circ} 20^{\prime} 45.1^{\prime \prime} \mathrm{E}, 19.07 .2009$, A. Ovchinnikov [ZIN].

DISTRIBUTION. Type locality: "Nord Ural: Voikar-Flufisysteme; Jakutien: Keedej-See, Berdzhastjach". Palaearctic: Mongolia, Russia (Khabarovsk, Primorye, Yamalia, Yakutia).

\section{Dolichopus bigeniculatus Parent, 1926}

MATERIAL EXAMINED. $20^{7}$, Spassk-Dalnii, $44.68^{\circ} \mathrm{N}$, 132.85 E, 15.06.2014, N. Vikhrev [ZMUM].

DISTRIBUTION. Type locality: China: Shanghai, "ZiKa-Wei” [=Xujiahui]. Palaearctic: China (Beijing, Henan, Shaanxi, Shandong), Japan, Russia (Primorye, Khabarovsk); Oriental: China (Sichuan, Anhui, Jiangsu, Zhejiang).

\section{Dolichopus cuneipennis Parent, 1926}

MATERIAL EXAMINED. $10^{7}$, Khanka Lake, $45.825^{\circ} \mathrm{N}$, 131.02 ${ }^{\circ} \mathrm{E}, 15-19.07 .2018$, N. Vikhrev [ZMUM].

DISTRIBUTION. Type locality: China: Tchen-Kiang, Zi-Ka-Wei [=Xujiahui, near Shanghai]. Palaearctic: China (Heilongjiang, Jilin, Shaanxi), Russia (Kuriles, Primorye); Oriental: China (Shanghai).

\section{Dolichopus hilaris Loew, 1862}

MATERIAL EXAMINED. $10^{7}$, Khanka Lake, $45.825^{\circ} \mathrm{N}$, $131.02^{\circ} \mathrm{E}, 15-19.07 .2018$, N. Vikhrev [ZMUM]; $10^{7}$, Andreevka, $42.7^{\circ} \mathrm{N}, 131.1^{\circ} \mathrm{E}, 26-31.07 .2018, \mathrm{~N}$. Vikhrev [ZMUM]; 10, $10^{\circ}$, Khankaisky distr., $6 \mathrm{~km} \mathrm{~N}$ Novokachalinsk, $45^{\circ} 09^{\prime} 31.8^{\prime \prime} \mathrm{N}$, 131 ${ }^{\circ} 59^{\prime} 58.2^{\prime \prime}$ E, 31.08-4.09.2019, Lake shore, Kosheleva [ZIN].

DISTRIBUTION. Type locality: Poland: Miedzyrecz. Palaearctic: Austria, Belarus, China(Heilongjiang, Xinjiang), Czech, Finland, France, Germany, Italy, Kazakhstan, Poland, Russia (Khabarovsk, Primorye, Saratov), Sweden, Tajikistan, Ukraine.

\section{Dolichopus leucopus Smirnov, 1948}

MATERIAL EXAMINED. $90^{\top}, 1$, , Oktyabrsky distr., $6.5 \mathrm{~km} \mathrm{~S}$ Chernyatino, $43^{\circ} 54^{\prime} 32.2^{\prime \prime} \mathrm{N}, 131^{\circ} 28^{\prime} 18.8^{\prime \prime} \mathrm{E}, 26-29.08 .2019$, Kosheleva [ZIN]; 1요 Pogranichnyi distr., Barabash-Levada, $44^{\circ} 45^{\prime} 54.7^{\prime \prime} \mathrm{N}, 131^{\circ} 24^{\prime} 59.6^{\prime \prime} \mathrm{E}, 5-9.09 .2019$, Kosheleva [ZIN]; 1ㅇ, Khankaisky distr., $6 \mathrm{~km}$ N Novokachalinsk, $45^{\circ} 09^{\prime} 31.8^{\prime \prime} \mathrm{N}$, $131^{\circ} 59^{\prime} 58.2^{\prime \prime} \mathrm{E}, 31.08-4.09 .2019$, Lake shore, Kosheleva [ZIN].

DISTRIBUTION. Type locality: Russia: Okeanskaya, near Vladivostok; Suchan; Kamen-Rybolov, Krivoy Klyuch, Gornotayozhnaya station. Palaearctic: Russia (Khabarovsk, Primorye).

\section{Dolichopus linearis Meigen, 1824}

MATERIAL EXAMINED. 107, Udege Legend National park, $45^{\circ} 46^{\prime} 05.2^{\prime \prime} \mathrm{N}, 135^{\circ} 20^{\prime} 45.1^{\prime \prime} \mathrm{E}, 16.07 .2009$, A. Ovchinnikov [ZIN]; $10^{7}, 10 \mathrm{~km}$ NE Vladivostok, $43.21^{\circ} \mathrm{N}, 132.07^{\circ} \mathrm{E}, 21-29.07 .2019$, E. Erofeeva [ZMUM].

DISTRIBUTION. Type locality: not given. Palaearctic: Austria, Belgium, China (Heilongiiang, Jilin, Beijing, Inner Mongolia, Gansu, Xinjiang, Qinghai), Czech Republic, Denmark, Estonia, Finland, France, Georgia, Germany, Hungary, Ireland, Italy, Kazakhstan, Latvia, Mongolia, Netherlands, Norway, Poland, Romania; Russia (Adygea, Altai Rep., Amur Region, Buryatia, Irkutsk, Kamchatka, Khabarovsk, Khanty-Mansi, Krasnodar, Krasnoyarsk, Leningrad, Magadan, Novgorod, Novosibirsk, Primorye, Pskov, Ryazan, Sakhalin, Tatarstan, Vologda, Voronezh, Yakutia), Slovakia, Sweden, Switzerland, UK.

\section{Dolichopus microstigma Stackelberg, 1930}

MATERIAL EXAMINED. $10^{\top}$, Andreevka, $42.64^{\circ} \mathrm{N}, 131.13^{\circ} \mathrm{E}$, 25-30.06.2014, N. Vikhrev [ZMUM].

DISTRIBUTION. Type locality: Russia: Ussuriisky (=Primorsky) Terr., Sedanka (originally published as "UssuriGebiet: Distr. Wladiwostok und Spassk). Palaearctic: Mongolia, Russia (Primorye, Sakhalin). 
Dolichopus nataliae Stackelberg, 1930

MATERIAL EXAMINED. $10^{\top}$, Andreevka, $42.64^{\circ} \mathrm{N}, 131.13^{\circ} \mathrm{E}$, 25-30.06.2014, N. Vikhrev [ZMUM].

DISTRIBUTION. Type locality: "Sibirie orientalis, prov. Austro-Ussuriensis, distr. Sutchaniensis; via Spassk-Jakovlevka, fl. Ugodzina, distr. Spasskensis, Tigrovaja". Palaearctic: Russia (Khabarovsk, Magadan, Primorye, Yakutia).

Dolichopus negrobovi Gosseries, 1989

MATERIAL EXAMINED. 107, Khasan distr., Troitsa Bay, 28.08.1984, V. Zlobin [ZIN].

DISTRIBUTION. Type locality: Russia: "Burjatskaja ASSR, Ustj-Bargusinskaja Tal, Jareehta". Palaearctic: Mongolia, Russia (Amur Region, Buryatia, Irkutsk, Kamchatka, Khabarovsk, Magadan, Primorye, Yakutia).

\section{Dolichopus nitidus Fallén, 1823}

MATERIAL EXAMINED. $10^{7}$, Udege Legend National park, $45^{\circ} 46^{\prime} 05.2^{\prime \prime} \mathrm{N}, 135^{\circ} 20^{\prime} 45.1^{\prime \prime} \mathrm{E}, 19.07 .2009$, A. Ovchinnikov [ZIN] $10^{7}$, Khanka Lake, $45.06^{\circ} \mathrm{N}, 131.99^{\circ} \mathrm{E}, 15-19.06 .2014$, N. Vikhrev [ZMUM]; $10^{7}$, Lotos Lake, $42.46^{\circ} \mathrm{N}, 130.64^{\circ} \mathrm{E}, 1-3.07 .2014, \mathrm{~N}$. Vikhrev [ZMUM]; $80^{2}$, Oktyabrsky distr., $6.5 \mathrm{~km} \mathrm{~S}$ Chernyatino, $43^{\circ} 54^{\prime} 32.2^{\prime \prime} \mathrm{N}, 131^{\circ} 28^{\prime} 18.8^{\prime \prime} \mathrm{E}, 26-29.08 .2019$, Kosheleva [ZIN].

DISTRIBUTION. Type locality: not given. Palaearctic: Austria, Belarus, Belgium, Bulgaria, China (Henan), Czech, Denmark, Estonia, Finland, France, Germany, Hungary, Ireland, ?Israel, Italy, Japan, Kazakhstan, Netherlands, Norway, Poland, Romania, Russia (Altai Rep., Karelia, Khabarovsk, Khanty-Mansi, Krasnodar, Moscow, Novgorod, Primorye, Ryazan, Tatarstan, Voronezh), Slovakia, Spain, Sweden, Switzerland, UK, Ukraine (Odessa); Oriental: China (Shanghai).

\section{Dolichopus plumipes (Scopoli, 1763)}

MATERIAL EXAMINED. $10^{7}$, Khanka Lake, $45.06^{\circ} \mathrm{N}$, 131.99² E, 15-19.06.2014, N. Vikhrev [ZMUM].

DISTRIBUTION. Type locality: Slovenia: "Carnioliae indigena". Mainly Holarctic species. Palaearctic China (Heilongjiang, Hebei, Henan, Shanxi, Inner Mongolia, Xinjiang, Qinghai, Xizang), East Russia (Chukotka, Irkutsk, Kamchatka, Khabarovsk, Koryakia, Novosibirsk, Primorye, Tomsk, Tyumen); Neotropical: Mexico; Oriental: India (Kashmir).

\section{Dolichopus plumitarsis Fallén, 1823}

MATERIAL EXAMINED. 10', Shkotovsky distr., Anisimovka, $43^{\circ} 10^{\prime} 07.0^{\prime \prime} \mathrm{N}, 132^{\circ} 46^{\prime} 11.3^{\prime \prime} \mathrm{E}, 11-13.08 .2019$, Kosheleva [ZIN].

DISTRIBUTION. Type locality: Sweden. Palaearctic: Austria, Belarus, Belgium, Bulgaria, China (Heilongjiang, Hebei, Beijing, Inner Mongolia, Xinjiang), Czech, Estonia, Finland, Germany, Italy, N Kazakhstan, Netherlands, Norway, Poland, Russia (Altai Rep., Buryatia, Chita, Commander Is., Karachai-Cherkessia, Irkutsk, Khabarovsk, Krasnodar, Krasnoyarsk, Kuriles, Moscow, Perm, Primorye, Sakhalin, Ural, Voronezh, Yakutia), Slovakia, Sweden, Switzerland, UK; Nearctic: Canada (Ontario), USA (Alaska).

\section{Dolichopus punctum Meigen, 1824}

MATERIAL EXAMINED. $20^{7}$, Daubikheza [=Sinegorka] River valley, Daubikhe [=Arsenyevka] tributary, 27.07.1962, Narchuk [ZIN]

DISTRIBUTION. Type locality: Germany: "Gegend von Berlin". Palaearctic: Austria, Finland, Germany, Poland, Russia (Khabarovsk, Leningrad, Moscow, Yakutia), Sweden. New for Primorye.

\section{Dolichopus rezvorum Stackelberg, 1930}

MATERIAL EXAMINED. 107, Bikin River, $22 \mathrm{~km}$ upstream Svetlovodnaya River mouth, 14.08.1980, V. Zlobin [ZIN]; $10^{7}$, Ussuri distr., Gornotaezhnoe, 16.06.1982, O. Ovchinnikova [ZIN];
$10^{7}$, Lazovsky Nat. Res., Korpad cordon, 8-10.07.2007, A. Ovchin-

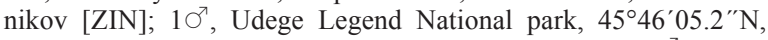
$135^{\circ} 20^{\prime} 45.1^{\prime \prime} \mathrm{E}, 20.07 .2009$, A. Ovchinnikov [ZIN]; $10^{7}$, Lotos Lake, $42.46^{\circ} \mathrm{N}, 130.64^{\circ} \mathrm{E}, 1-3.07 .2014, \mathrm{~N}$. Vikhrev [ZMUM]; $10^{\mathrm{T}}$, Shkotovsky distr., Anisimovka, $43^{\circ} 10^{\prime} 07.0^{\prime \prime} \mathrm{N}, 132^{\circ} 46^{\prime} 11.3^{\prime \prime} \mathrm{E}, 11-$ 13.08.2019, Kosheleva [ZIN]; $10^{7}, 10 \mathrm{~km}$ NE Vladivostok, $43.21^{\circ} \mathrm{N}$, 132.07 E, 21-29.07.2019, E. Erofeeva [ZMUM].

DISTRIBUTION. Type locality: Russia: Tigrovaja, Sedanka, Spassk-Yakovlevka, river Ugodinza. Palaearctic: Mongolia, Russia (Krasnoyarsk, Khabarovsk, Primorye).

\section{Dolichopus ringdahli Stackelberg, 1930}

MATERIAL EXAMINED. $10^{7}$, Ussuri distr., Gornotaezhnoe, 5.09.1980, V. Zlobin [ZIN].

DISTRIBUTION. Type locality: "Kreis Jakutsk: KeedejSee; Sud-Ussuri-Gebiet: Tigrovaya, Kreis Sutshan”. Palaearctic: Russia (Buryatia, Primorye, Yakutia), China (Jilin).

\section{Dolichopus robustus Stackelberg, 1928}

MATERIAL EXAMINED. $10^{7}$, Kamenushka, $43.62^{\circ} \mathrm{N}$, 132.23 ${ }^{\circ}$ E, 22-24.06.2014, N. Vikhrev [ZMUM].

DISTRIBUTION. Type locality: "Siberiae orientalis, prov. Austro-Ussuriensis propepagum Tigrovaja distr. Sutshanicus; Sedanka prope Vladivostok; in ripis fluminis Ugodinza, via Spassk-Jakovlevka, distr. Spasskensis". Palaearctic: Russia (Altai Rep., Amur Region, Irkutsk, Kamchatka, Khabarovsk, Krasnoyarsk, Moscow, Primorye, Sayan Mnts., Yakutia), China (Shandong).

\section{Dolichopus setimanus Smirnov, 1948}

MATERIAL EXAMINED. 107, Ussuri distr., Gornotaezhnoe, 31.07.1980, Ovchinnikova [ZIN]. 407, Ussuri distr., Gornotaezhnoe, 8, 9, 26.08.1980, Afanasyeva [ZIN].

DISTRIBUTION. Type locality: Okeanskaya, near Vladivostosk. Palaearctic: Russia (Amur Region, Chita, Khabarovsk, Primorye, Sakhalin, Kuriles)

\section{Dolichopus uniseta Stackelberg, 1929}

MATERIAL EXAMINED. $10^{7}$, Khanka Lake, $45.06^{\circ} \mathrm{N}$, 131.99 ${ }^{\circ}$ E, 15-19.06.2014, N. Vikhrev [ZMUM]; 10 $0^{2}$, Lotos Lake, $42.46^{\circ} \mathrm{N}, 130.64^{\circ} \mathrm{E}, 1-3.07 .2014, \mathrm{~N}$. Vikhrev [ZMUM]; $20^{\prime}$, Lazovsky distr., Preobrazhenie, Tasovaya, 42 $53^{\prime} 44.4^{\prime \prime} \mathrm{N}$, $133^{\circ} 57^{\prime} 13.4^{\prime \prime E}, 20-21.08 .2019$, Kosheleva [ZIN]; 1007 , 3ㅇ, Shko-

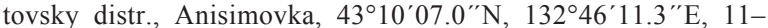
13.08.2019, Kosheleva [ZIN]

DISTRIBUTION. Type locality: Russia: "Kreis Jakutsk: Olom; Abyj, ungefahr $60^{\circ} 50^{\prime}$ nordlicher Breite und $130^{\circ}$ Ostlicher Lange zwischen der Lena und Amga, Amginiskaja Sloboda, amlinken Ufer des Flukes Amga; Sud-Ussuri-Gebiet: Jakovlevka, Kreis Spassk". Palaearctic: Russia (Khabarovsk, Primorye, Yakutia), China (Heilongjiang, Hebei, Beijing, Shaanxi).

Dolichopus ussuriensis Stackelberg, 1930

MATERIAL EXAMINED. 20', 1ㅇ, Ussuri distr., Gornotaezhnoe, 3.06.1982, Ovchinnikova [ZIN].

DISTRIBUTION. Type locality: Majkhe [=Shtykovo], near Shkotov, Tigrovaya, Spassk-Yakovlevka, river Ugodinza. Palaearctic: Russia (Amur Region, Khabarovsk, Primorye).

\section{Dolichopus varians Smirnov, 1948}

MATERIAL EXAMINED. 10', Oktyabrsky distr., $6.5 \mathrm{~km} \mathrm{~S}$ Chernyatino, $43^{\circ} 54^{\prime} 32.2^{\prime \prime} \mathrm{N}, 131^{\circ} 28^{\prime} 18.8^{\prime \prime} \mathrm{E}, 26-29.08 .2019$, Kosheleva [ZIN]; $20^{7}$, Lazovsky distr., Preobrazhenie,Tasovaya, $42^{\circ} 53^{\prime} 44.4^{\prime \prime} \mathrm{N}, 133^{\circ} 57^{\prime} 13.4^{\prime \prime} \mathrm{E}, 20-21.08 .2019$, Kosheleva [ZIN];

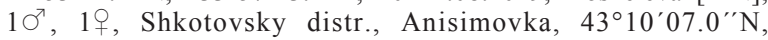
13246'11.3"E, 11-13.08.2019, Kosheleva [ZIN].

DISTRIBUTION. Type locality: Russia: near Vladivostok, Okeanskaya, Sedanka, Suchan - Sergeevka. Palaearctic: Russia (Kamchatka, Khabarovsk, Primorye), Korea. 
Dolichopus xanthopyga Stackelberg, 1930

MATERIAL EXAMINED. $10^{7}$, Khanka Lake, $45.825^{\circ} \mathrm{N}$, $131.02^{\circ} \mathrm{E}, 15-19.07 .2018$, N. Vikhrev [ZMUM]; 30 ${ }^{7}$, Khankaisky distr., $6 \mathrm{~km}$ N Novokachalinsk, $45^{\circ} 09^{\prime} 31.8^{\prime \prime} \mathrm{N}, 131^{\circ} 59^{\prime} 58.2^{\prime \prime} \mathrm{E}, 31.08$ 4.09.2019, Lake shore, Kosheleva [ZIN].

DISTRIBUTION. Type locality: Russia: "Siberie orientalis prov. Spasskensis litis meridionalis locus, Chanka prope pagum Staraja Devitza, ad ostium fluminis Lefu, promontorium Rjabokonj”. Palaearctic: Russia (Khabarovsk, Primorye), China (Heilongjiang).

\section{Genus Gymnopternus Loew, 1857}

NOTES. Until 2005 the genus had long been supposed to be a synonym of Hercostomus in Russian literature with none reported species from the Russian Far East. Negrobov et al. [2016] transferred Hercostomus ussurianus Stackelberg, 1933 to Gymnopternus. Two species of the same genus are transferred to Gymnopternus below. At least two more different species are found in ZMUM collection from the South of the Primorsky Territory; these species need further study. More than forty species are known from China.

Gymnopternus daubichensis (Stackelberg, 1933), comb.n.

=Hercostomus daubichensis Stackelberg, 1933: 121 (in key) (descr.: ibid. 1934: 140).

MATERIAL EXAMINED. $10^{7}$, Ussuri distr., Gornotaezhnoe, 20-30.06.2007, A. Ovchinnikov [ZIN]; 10 , Gornye Klyuchi, $45.25^{\circ} \mathrm{N}, 133.50^{\circ} \mathrm{E}, 6-7.07 .2014, \mathrm{~N}$. Vikhrev [ZMUM]; $10^{7}$, Khanka Lake, $45.825^{\circ} \mathrm{N}, 131.02^{\circ} \mathrm{E}, 15-19.07 .2018$, N. Vikhrev [ZMUM].

DISTRIBUTION. Type locality: "Ussuri-Gebiet, Dorf Jakovlevka, Distrikt Spassk”. Palaearctic: Russia (Primorye).

\section{Gymnopternus nemorum}

(Smirnov et Negrobov), 1977, comb.n.

=Hercostomus nemorum Smirnov et Negrobov, 1977: 89.

MATERIAL EXAMINED. $10^{7}$, Andreevka, $42.7^{\circ} \mathrm{N}, 131.1^{\circ} \mathrm{E}$ 26-31.07.2018, N. Vikhrev [ZMUM].

DISTRIBUTION. Type locality: Partisansk. Palaearctic: Russia (Primorye)

Gymnopternus ussurianus (Stackelberg, 1933)

MATERIAL EXAMINED. 19, Kamenushka, 29.08.1978, V.Zlobin [ZIN]; 60', 1으, Ussuri distr., Gornotaezhnoe, 15.viii, 5.09.1980, V. Zlobin [ZIN]; 40', Khasan distr., Troitsa Bay, 27-28.08.1984, V. Zlobin [ZIN]; 107, 19, Kamenushka, 26.06.1980, V. Zlobin [ZIN]; $10^{7}$, Kedrovaya Pad Reserve, 30.08.1980, V. Zlobin [ZIN]; 20', Shkotovsky distr., Anisimovka, 20.07.2004, V. Zlobin [ZIN]; 10', Udege Legend National park, $45^{\circ} 46^{\prime} 05.2^{\prime \prime} \mathrm{N}, 135^{\circ} 20^{\prime} 45.1^{\prime \prime} \mathrm{E}, 17.07 .2009$, A Ovchinnikov [ZIN]; $10^{\top}$, Anuchino env., $43.95^{\circ} \mathrm{N}, 133.05^{\circ} \mathrm{E}, 20$ 21.06 .2014 , N. Vikhrev [ZMUM]; $20^{7}$, Khanka Lake, $45.06^{\circ} \mathrm{N}$, $131.99^{\circ} \mathrm{E}, 15-19.06 .2014$, N. Vikhrev [ZMUM]; 10", Gornye Klyuchi, $45.25^{\circ} \mathrm{N}, 133.50^{\circ} \mathrm{E}, 6-7.07 .2014$, N. Vikhrev [ZMUM]; 30, $\mathrm{An}-$ dreevka, $42.7^{\circ} \mathrm{N}, 131.1^{\circ} \mathrm{E}, 26-31.07 .2018$, N. Vikhrev [ZMUM]; 307, Ryazanovka River, $46.827^{\circ} \mathrm{N}, 131.23^{\circ} \mathrm{E}, 1.08 .2018$, N. Vikhrev [ZMUM]; $20^{7}$, Khanka Lake, $45.06^{\circ} \mathrm{N}, 131.99^{\circ} \mathrm{E}, 4-6.07 .2014, \mathrm{~N}$ Vikhrev [ZMUM]; $10^{7}$, Khanka Lake, $45.825^{\circ} \mathrm{N}, 131.02^{\circ} \mathrm{E}, 15-$ 19.07.2018, N. Vikhrev [ZMUM]; $10^{7}, 10 \mathrm{~km}$ NE Vladivostok, $43.21^{\circ} \mathrm{N}$, $132.07^{\circ} \mathrm{E}, 21-29.07 .2019$, E. Erofeeva [ZMUM]; 30', Oktyabrsky distr., $6.5 \mathrm{~km} \mathrm{~S}$ Chernyatino, $43^{\circ} 54^{\prime} 32.2^{\prime \prime} \mathrm{N}, 131^{\circ} 28^{\prime} 18.8^{\prime \prime} \mathrm{E}, 26$ 29.08.2019, Kosheleva [ZIN]; 3807, Lazovsky distr., Preobrazhenie, Tasovaya, 42 ${ }^{\circ} 53^{\prime} 44.4^{\prime \prime} \mathrm{N}, 133^{\circ} 57^{\prime} 13.4^{\prime \prime} \mathrm{E}, 20-21.08 .2019$, Kosheleva [ZIN]; $10^{7}, 1$ 을 Shkotovsky distr., Anisimovka, $43^{\circ} 10^{\prime} 07.0^{\prime \prime} \mathrm{N}$, $132^{\circ} 46^{\prime} 11.3^{\prime \prime} \mathrm{E}, 11-13.08 .2019$, Kosheleva [ZIN].

DISTRIBUTION. Type locality: Russia: "Ussuri-Gebiet, Tigrovaja, Sutshan-Distr., Jakovlevka, Spassk-Distr., Baddargin bei Wladiwostok, Rjabokonj am Chanka-See". Palaearctic: Japan, Russia (Amur Region, Khabarovsk, Primorye).
Genus Hercostomus Loew, 1857

NOTES. One male collected from environs of Vladivostok belongs most probably to an undescribed species; it needs further study, because more than 200 species are known currently from China.

\section{Hercostomus sviridovae Negrobov et Chalaya, 1987}

MATERIAL EXAMINED. 107, 20 km SE Ussuriisk, 31.08.1978, Kasparyan [ZIN].

DISTRIBUTION. Type locality: Primorye, Bikin river. Palaearctic: Russia (Buryatia, Chita, Primorye).

\section{Genus Hydrophorus Fallén, 1823}

NOTES. One male collected from environs of Khanka Lake belongs most probably to an undescribed species; it needs further study. Two species of the genus are known from Primorye and nine species from China.

Genus Medetera Fischer von Waldheim, 1819

Medetera flavigena Masunaga et Saigusa, 1998

MATERIAL EXAMINED. $10^{7}$, Kamenushka, $43.62^{\circ} \mathrm{N}$, 132.23ํㅡ, 22-24.vi.2014, N. Vikhrev [ZMUM].

DISTRIBUTION. Type locality: Japan: Honshu, Ojiya. Palaearctic: Japan. New for Russia and Primorye.

Genus Micromorphus Mik, 1878

Micromorphus jinshanensis (Wang, Yang et Grootaert, 2009), comb.n.

=Nepalomyia jinshanensis Wang, Yang et Grootaert, 2009: 44 .

MATERIAL EXAMINED. $80^{7}, 2+$, Khankaisky distr., $6 \mathrm{~km} \mathrm{~N}$ Novokachalinsk, $45^{\circ} 09^{\prime} 31.8^{\prime \prime} \mathrm{N}, 131^{\circ} 59^{\prime} 58.2^{\prime \prime} \mathrm{E}, 31.08-4.09 .2019$, Lake shore, Kosheleva [ZIN].

NOTES. The species was described by two males [Wang et al., 2009]. The holotype was found in environs of Beijing, while the paratype was collected from the Oriental Sichuan province (Qingchengshan mountain). The description contains a mixture of Nepalomyia and Micromorphus generic concepts. Nevertheless, the figures of antenna and hypopygium provided by the authors clearly testify that the Nepalomyia jinshanensis must be recombined with the genus Micromorphus. The Russian material examined is identical morphologically with those figures. However, despite the description, our specimens has no acrostichal setae, hind basitarsus has indistinct basal spur in males, hypandrium is simple, without deep V-shaped apical incision, but forming acute angle with simple phallus. It is highly likely that the description of $N$. jinshanensis belongs to the paratype representing a different species of mainly Oriental Nepalomyia, while the figures belong to the holotype representing a species of Micromorphus. On the other hand, Micromorphus ellampus Wei, 2006 and M. heterophalla Wei et Yang, 2007, both described from the Oriental Guizhou province and bearing acrostichals on the mesonotum [Wei, 2006; Wei, Yang, 2007], belong probably to another genus.

DISTRIBUTION. Type locality: Beijing: Jinshan mountain. Palearctic: China (Beijing); ?Oriental: China (Sichuan). New for Russia. The genus is new for Primorye.

\section{Genus Neurigona Rondani, 1856}

NOTES. The Neurigona fauna of Primorye contains five species. The Chinese fauna includes 31 species.

\section{Neurigona micropyga Negrobov, 1987}

MATERIAL EXAMINED. 10 , Khasan District, $3 \mathrm{~km}$ W Ryazanovka, Malaise trap, 16.07.1992, P. Lindskog \& A. Nilsson leg. (Swedish Museum of Natural History, Stockholm).

DISTRIBUTION. Type locality: Kuril Is. Palaearctic: China (Henan), Japan, Russia (Kuriles). New for Primorye. 
Neurigona pullata Negrobov et Fursov, 1988

MATERIAL EXAMINED. $1 \sigma^{7}$, South Kamenushka, 4.06.1984, A. Shatalkin [ZMUM]

DISTRIBUTION. Type locality: Primorye, Kedrovaya Pad Reserve. Palaearctic: Russia (Amur Region, Primorye, Sakhalin).

\section{Genus Poecilobothrus Mik, 1878}

NOTES. Until now the genus Poecilobothrus, belonging to the subfamily Dolichopodinae, had long been supposed to be Mediterranean in distribution, with 16 known species [Grichanov, 2017] occurring in the West Palaearctic countries with relatively mild climate, from southern Scandinavia in the North, to North Africa and Central and Middle Asia (Iran, Tajikistan and Uzbekistan) in the South [I raise here Poecilobothrus flavifemoratus Grichanov et Tonguç, 2010, subspecies of P. varicoloris (Becker, 1917), to a species level (stat.n.)]. With new re-combinations in this paper (see below) the genus is distributed in the Russian Prymorye and all over China in addition to West Palaearctic countries, numbering totally 31 valid species. See also comments under Poecilobothrus pterostichoides.

\section{Poecilobothrus flaveolus}

(Negrobov et Chalaya, 1987), comb.n.

=Hercostomus flaveolus Negrobov et Chalaya, 1987: 45 .

=Poecilobothrus arcticus (Yang, 1996), comb.n.

=Hercostomus arcticus Yang, 1996: 235 (in subgenus Hercostomus). Type locality: China: Heilongjiang, Ningan.

MATERIAL EXAMINED. 107, Bikin River, $22 \mathrm{~km}$ upstream Svetlovodnaya River mouth, 19.07.1980, V. Zlobin [ZIN]; $10^{\text {T}}$ Lazovsky distr., Preobrazhenie, Tasovaya, 42 $53^{\prime} 44.4^{\prime \prime} \mathrm{N}$ $133^{\circ} 57^{\prime} 13.4^{\prime \prime} \mathrm{E}, 20-21.08 .2019$, Kosheleva [ZIN]; 207, 1, Oktyabrsky distr., $6.5 \mathrm{~km} \mathrm{~S}$ Chernyatino, 435' $32.2^{\prime \prime} \mathrm{N}, 131^{\circ} 28^{\prime} 18.8^{\prime \prime} \mathrm{E}$ 26-29.08.2019, Kosheleva [ZIN]; 6 $\sigma^{7}, 19$, Shkotovsky distr., Anisi-

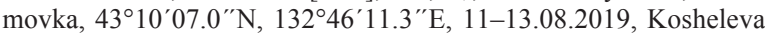
[ZIN].

NOTES. Hercostomus arcticus was synonymized with H. flaveolus by Negrobov et al. [2016]. See also comments under Poecilobothrus pterostichoides.

DISTRIBUTION. Type locality: Primorye, Spassky distr., Nakhimovka. Palaearctic: China (Beijing, Heilongjiang, Shaanxi), Japan, Russia (Amur Region, Khabarovsk, Primorye); Oriental: China (Hunan).

\section{Poecilobothrus pterostichoides (Stackelberg, 1934), comb.n.}

=Hercostomus pterostichoides Stackelberg, 1934:118 (in key) (descr. ibid.: 166)

=Poecilobothrus brevipilosus (Yang et Saigusa, 2002), comb.

n.

=Hercostomus brevipilosus Yang et Saigusa, 2002: 65 (in subgenus Hercostomus), syn.n. Type locality: China: Shaanxi, Fuping.

MATERIAL EXAMINED. $10^{7}, 1$, , Shkotovsky distr., Anisi-

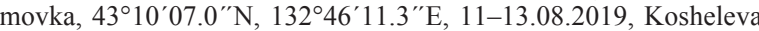
[ZIN]; $80^{7}, 10 \mathrm{~km}$ NE Vladivostok, $43.21^{\circ} \mathrm{N}, 132.07^{\circ} \mathrm{E}, 21-$ 29.07.2019, E. Erofeeva [ZMUM]

DISTRIBUTION. Type locality: "Ussuri-Gebiet, bei der Station Tigrovaja, Sutshan" [=Ussuriiskaya oblast, near Tigrovaya station, Suchan; now Primorsky krai, Tigrovoi village, Partizansk]. Palaearctic: China (Beijing, Shaanxi), Russia (Primorye).

NOTES. The species was described by males and females collected on 3-4 August 1927 from the Tigrovoi locality [Stackelberg, 1934]. It was never found later again [Negrobov et al., 2013]. New material collected from the Anisimovka village, which is very close (about $5 \mathrm{~km}$ ) to the type locality of Hercostomus pterostichoides, stimulated the reexamination of descriptions of similar Chinese species of the genus [Yang et al., 2011]. As it turned out, H. pterostichoides belongs to the Hercostomus cyaneculus group containing 14 Chinese species, of which none corresponds to the nominotypical Hercostomus longiventris lineage [as defined by Brooks, 2005]. According to the generic key of latter author, the Hercostomus cyaneculus group as a whole must be associated with the genus Poecilobothrus Mik, 1878. This genus can be distinguished [Brooks, 2005] by the possession of a distinct dark metallic spot above the notopleuron, and one strong posterior to posteroventral preapical seta on the mid femur. Males are further distinguished by their distinctive postgonite and short, conical, slightly dorsoventrally flattened hypandrium, fused to epandrium laterally near basoventral epandrial lobe. Females are further distinguished by the possession of an inner, medial pair of spines on tergite 10 . Some species [e.g., P. regalis (Meigen, 1824), P. aberrans (Loew, 1871)] have one dorsal seta on hind basitarsus. Arista-like stylus is more or less strongly pubescent or even plumose (P. aberrans) that was noted by Stackelberg [1934] in his description of $H$. pterostichoides (the specific name itself is related with an unjustified emendation of the generic name Pterostylus, now synonym of Poecilobothrus).

An examination of available Chinese material in ZIN collection for three species from the Hercostomus cyaneculus group (identified by Ding Yang, Chinese Agricultural University, Beijing) in addition to $H$. flaveolus and $H$. pterostichoides has confirmed the presence of those characters listed by Brooks [2005]. Moreover, distributed in Palaearctic China Hercostomus brevipilosus is identical with $H$. pterostichoides. So, the following new combinations are here proposed:

Poecilobothrus brunus (Wei, 1997), comb.n. (Hercostomus);

Poecilobothrus cucullus (Wei, 1997), comb.n.(Hercostomus); Poecilobothrus cyaneculus (Wei, 1997), comb.n. (Hercostomus);

Poecilobothrus lii (Yang, 1996), comb.n. (Hercostomus);

Poecilobothrus longipilosus (Yang et Saigusa, 2001), comb.n. (Hercostomus);

Poecilobothrus luchunensis (Yang et Saigusa, 2001), comb.n. (Hercostomus);

Poecilobothrus mentougouensis (Zhang, Yang et Grootaert, 2003), comb.n. (Hercostomus);

Poecilobothrus palustrus (Wei, 2006), comb.n. (Hercostomus);

Poecilobothrus potanini (Stackelberg, 1934), comb.n. (Hercostomus);

Poecilobothrus saetosus (Yang et Saigusa, 2002), comb.n. (Hercostomus);

Poecilobothrus singularis (Yang et Saigusa, 2001), comb.n. (Hercostomus);

Poecilobothrus zhejiangensis (Yang, 1996), comb.n. (Hercostomus).

Genus Rhaphium Meigen, 1803

NOTES. One more male collected from Anreeevka belongs most probably to an undescribed species.

Rhaphium dispar Coquillett, 1898

MATERIAL EXAMINED. $10^{7}$, Khanka Lake, $45.06^{\circ} \mathrm{N}$, 131.99 ${ }^{\circ}$ E, 15-19.06.2014, N. Vikhrev [ZMUM].

DISTRIBUTION. Type locality: Japan. Palaearctic: Russia (Kamchatka, Magadan, Primorye), Japan; Oriental: China (Guizhou, Sichuan, Taiwan, Zhejiang). 
Rhaphium flavilabre Negrobov, 1979

MATERIAL EXAMINED. $10^{\top}$, Andreevka, $42.64^{\circ} \mathrm{N}$, $131.13^{\circ} \mathrm{E}, 25-30.06 .2014$, N. Vikhrev [ZMUM]; $10^{7}$, Khasan distr., Troitsa Bay, 28.08.1984, V. Zlobin [ZIN].

DISTRIBUTION. Type locality: Primorye, KomarovoZapovednoe, Nature Reserve. Palaearctic: Russia (Khabarovsk, Primorye).

\section{Rhaphium micans (Meigen, 1824)}

MATERIAL EXAMINED. $10^{7}$, Khanka Lake, $45.06^{\circ} \mathrm{N}$, 131.99 ${ }^{\circ} \mathrm{E}, 15-19.06 .2014$, N. Vikhrev [ZMUM].

DISTRIBUTION. Type locality: Germany: "Hamburg". Palaearctic: Abkhazia, Austria, Azerbaijan, Belarus, Belgium, Bulgaria, China, Czech, Finland, France, Germany, Hungary, Italy, Latvia, Netherlands, Norway, Poland, Romania, Russia (Adygea, Astrakhan, Kabardino-Balkaria, Karachai-Cherkessia, Karelia, Khabarovsk, Krasnodar, Krasnoyarsk, Leningrad, Primorye, Pskov, Rostov, Ryazan, Voronezh), Serbia, Slovakia, Spain, Sweden, Switzerland, Tajikistan, Turkey, UK.

\section{Rhaphium nasutum (Fallén, 1823)}

MATERIAL EXAMINED. $10^{7}$, Khanka Lake, $45.06^{\circ} \mathrm{N}$, 131.99 ${ }^{\circ}$ E, 15-19.06.2014, N. Vikhrev [ZMUM].

DISTRIBUTION. Type locality: Sweden: "Svecia meridionali”. Nearctic: Canada, USA; Palaearctic: Austria, Belarus, Belgium, Czech, Denmark, Finland, France, Germany, Hungary, Kazakhstan, Netherlands, Norway, Poland, Romania, Russia (Bryansk, Krasnoyarsk, Leningrad, Moscow, Primorye, Ryazan, Voronezh, Yakutia), Slovakia, Sweden, UK.

Genus Suschania Negrobov, 2003

Suschania stackelbergi Negrobov, 2003

MATERIAL EXAMINED. 107, Ussuri distr., Gornotaezhnoe, 15.07.1980, V. Zlobin [ZIN].

DISTRIBUTION. Type locality: Primorsky Terr., Suschan, Tigrovaya. Palaearctic: Russia (Primorye).

Genus Syntormon Loew, 1857

Syntormon flexibilis Becker, 1922

MATERIAL EXAMINED. $10^{\top}$, Andreevka, $42.64^{\circ} \mathrm{N}, 131.13^{\circ} \mathrm{E}$, 25-30.06.2014, N. Vikhrev [ZMUM].

DISTRIBUTION. Type locality: China: Taiwan: Taihorka; Anping; Tainan. Afrotropical: St Helena; Australasian: Australia, French Polynesia, Japan (Bonin I.), New Caledonia, Tonga, USA (Hawaii); Nearctic: western Canada, northwestern USA; Oriental: China (Guangdong, Guizhou, Shanghai, Taiwan, Zhejiang); Palaearctic: China (Hebei, Jiangsu), Japan, Russia (Primorye).

Syntormon monochaetus Negrobov, 1975

MATERIAL EXAMINED. $10^{7}$, Lotos Lake, $42.46^{\circ} \mathrm{N}, 130.64^{\circ} \mathrm{E}$, 1-3.07.2014, N. Vikhrev [ZMUM].

DISTRIBUTION. Type locality: Maritime Territory, Primorye, Yakovlevka. Palaearctic: Russia (Buryatia, Khabarovsk, Primorye).

Genus Thinophilus Wahlberg, 1844

Thinophilus longipilus Negrobov, 1971

MATERIAL EXAMINED. $10^{7}$, Khanka Lake, $45.06^{\circ} \mathrm{N}$, $131.99^{\circ} \mathrm{E}, 15-19.06 .2014$, N. Vikhrev [ZMUM]; $10^{7}$, Luchegorsk, $46.47^{\circ} \mathrm{N}, 134.32^{\circ} \mathrm{E}, 22-24.06 .2014$, N. Vikhrev [ZMUM].

DISTRIBUTION. Type locality: Maritime Territory, Khanka Lake, Kamen-Rybolov. Palaearctic: Japan, Russia (Primorye).
Acknowledgements. The author is sincerely grateful to Drs. I.V. Shamshev (ZIN), N.E. Vikhrev and A.L. Ozerov (ZMUM), for their kindness in providing specimens for study. The work was funded by RFBR and NSFC according to the research project No 20-54-53005. The comparative analysis of morphological characters was performed within the Program for Basic Scientific Research of the Government of the Russian Federation, and supported by the AllRussian Institute of Plant Protection project No 06652020-0014.

\section{References}

Brooks S.E. 2005. Systematics and phylogeny of the Dolichopodinae (Diptera: Dolichopodidae) // Zootaxa. Vol.857. P.1158.

Grichanov I.Ya. 2017. Alphabetic list of generic and specific names of predatory flies of the epifamily Dolichopodoidae (Diptera). 2nd ed. Saint Petersburg: All-Russian Research Institute of Plant Protection. 563 pp. DOI: 10.5281/zenodo. 884863 .

Grichanov I.Ya., Tonguc A. 2010. New contribution to the Turkish Dolichopodidae (Diptera) fauna and taxonomy // International Journal of Dipterological Research. Vol.21. No.3. P.225-229.

Kiselev A.N., Kudryavtseva E.P. 1998. [Vegetation] // Nazdratenko E.I. (ed.). Atlas Primorskogo kraya. Vladivostok: Nauka. P.4-5 [in Russian].

Negrobov O.P., Chalaya O.N. 1987. [New data on the genus Hercostomus Loew (Dolichopodidae, Diptera)] // Nauchnye doklady vysshei shkoly, Biologicheskie nauki. No.5. P.42-46 [in Russian].

Negrobov O.P., Kumazawa T., Tago T., Sato M. 2016. New species of Hercostomus Loew, 1857 (Dolichopodidae, Diptera) from Japan // Zootaxa. Vol.4158. No.1. P.65-80.

Negrobov O.P., Selivanova O.V., Maslova O.O., Chursina M.A. 2013. Check-list of predatory flies of the family Dolichopodidae (Diptera) in the fauna of Russia // Grichanov I.Ya., Negrobov O.P. (eds.). Fauna and taxonomy of Dolichopodidae (Diptera). Collection of papers. St.Petersburg: VIZR RAAS (Plant Protection News Supplements ISSN 1815-3682). P.47-93.

Smirnov E.S., Negrobov O.P. 1977. [A new species of Hercostomus Lw. (Diptera, Dolichopodidae) from Primorye] // Cherepanov A.I. (ed.) Taksony fauny Sibiri. Novosibirsk: Nauka. P.89-91 [in Russian].

Stackelberg A.A. 1933. Dolichopodidae // E. Lindner (Hrsg.). Die Fliegen der Paläarktischen Region. Bd.IV/5. Teil 29. Lfg.71. S.65-128.

Stackelberg A.A. 1934. Dolichopodidae // E. Lindner (Hrsg.). Die Fliegen der Paläarktischen Region. Bd.IV/5. Teil 29. Lfg.82. S.129-176.

Wang M., Yang D., Grootaert P. 2009. New species of Nepalomyia from China (Diptera: Dolichopodidae) // Zootaxa. Vol.2162. P.37-49.

Wei L. 2006. [Dolichopodidae] // Li Z.Z., Jin D.C. (eds.). Insects from Fanjingshan Landscape. Guiyang: Guizhou Science and Technology Publishing House. P.468-502 [in Chinese with English summary].

Wei L.M., Yang Z.H. 2007. [Dolichopodidae] // Li Z.J., Yang M.F., Jin D.C. (eds.). Insects from Leigongshan Landscape. Guiyang: Guizhou Science and Technology Publishing House. P.561-587 [in Chinese, with English summary].

Yang D., Zhang L., Wang M., Zhu Y. 2011. Fauna Sinica, Insecta, Volume 53, Dolichopodidae. Beijing: Science Press. 1912 p. [in Chinese, with English summary].

Yang D., Zhang L.L., Zhang K.Y. 2018. Species Catalogue of China. Vol.2. Animals, Insecta (VI), Diptera (2), Orthorrhaphous Brachycera. Beijing: Science Press. 387 p.

Yang D., Saigusa T. 2002. The species of Hercostomus from the Qinling Mountains of Shaanxi, China (Diptera, Empidoidea, Dolichopodidae)//Deutsche Entomologische Zeitschrift. Vol.49. No.1. P.61-88. 